\title{
Light-Harvesting Supramolecular Polymers: Energy Transfer to Various Polyaromatic Acceptors
}

\author{
Jovana Jevric, Simon M. Langenegger and Robert Häner*
}

\author{
Jovana Jevric, Dr. Simon Matthias Langenegger and Prof. Dr. Robert Häner \\ Department of Chemistry and Biochemistry \\ University of Bern \\ Freiestrasse 3, 3012 Bern \\ E-Mail: robert.haener@dcb.unibe.ch \\ https://haener.dcb.unibe.ch
}

Supporting information for this article is given via a link at the end of the document.

\begin{abstract}
A supramolecular light-harvesting antenna consisting of 3,6-disubstituted phosphodiester-linked phenanthrene trimers (donors) was doped with different charged and uncharged polyaromatic and heteroaromatic acceptor chromophores. Excitation of the phenanthrene moieties is followed by energy transfer to the acceptor molecules and radiative relaxation. The self-assembled fiber structure of the phenanthrene trimers is not altered by the dopant, as verified by atomic force microscopy (AFM). Among several neutral and cationic acceptor chromophores, benzo(a)pyrene emerged as one of the highest fluorescence quantum yield, reaching $31 \%$ at a chromophore/phenanthrene ratio of $12 \mathrm{~mol} \%$. The energy transfer is probably a combination of Förster resonance energy transfer (FRET) and a coherent energy transfer mechanism.
\end{abstract}

The interest in artificial light-harvesting complexes (LHCs) has significantly increased in recent years. ${ }^{[1]}$ Efficient energy transfer is favored by a high degree of structural organization. Supramolecular polymers ${ }^{[2,3]}$ have been used as versatile scaffolds to arrange donor and acceptor building blocks in artificial LHCs. Along with several practical advantage ${ }^{[1 f, 2 a, 4]}$ the dynamic nature of supramolecular polymers renders them also amenable to easy modification, e.g. by adding different chromophores as acceptors in artificial LHCs. ${ }^{[16,5]}$ In previous work, we have demonstrated the construction of efficient, supramolecular $1 \mathrm{D}$ and $2 \mathrm{D}$ light-harvesting systems. ${ }^{[3,5 d, 6]}$ In one example, supramolecular light-harvesting fibers were formed by self-assembly of 3,6-dialkynyl-linked phenanthrene trimers. ${ }^{[5 d]}$ The fibers were doped with a tetramer consisting of three phenanthrenes and a pyrene moiety on one end. After excitation of the phenanthrenes, energy was transferred to the pyrene. After the addition of $1.5 \%$ pyrene, the fluorescence quantum yield reached $50 \% .{ }^{[5 d]}$ In another approach, a 2,7-dialkynyllinked phenanthrene trimer was synthesized, which selfassembles in aqueous media into nanotubes. ${ }^{[3 b]}$ These nanotubes were doped with a trimer consisting of two phenanthrenes and a pyrene molecule in the middle linked by phosphodiester bonds. The phenanthrenes in the nanotubes act as a donor and the pyrene as an acceptor. The doped nanotubes exhibit a fluorescence quantum yield of $23 \%$ at a concentration of $7 \%$ pyrene. It was shown that an efficient energy transfer occurs along the nanotubes. ${ }^{[3 \mathrm{~b}]}$ Both examples made use of acceptor chromophores that were covalently attached to oligomeric donor molecules in order to ensure integration of the acceptor into the supramolecular polymer.
Herein, we demonstrate that supramolecular phenanthrene (Phe) polymers also form light-harvesting systems with noncovalently linked acceptor molecules.
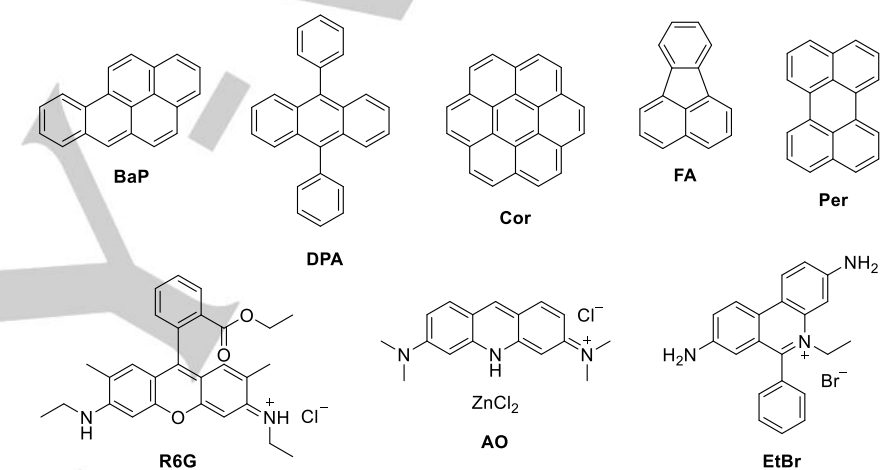

Figure 1. Chemical structures of the tested chromophores: benzo(a)pyrene (BaP), 9,10-diphenylanthracene (DAP), coronene (Cor), fluoranthene (FA), perylene (Per), rhodamine 6G (R6G), acridine orange (AO), ethidium bromide (EtBr).

Different acceptor molecules (Figure 1) were investigated for their effect on energy transfer in supramolecular polymer fibers assembled from Phe trimer $\mathbf{A}$ (Figure 2). Oligomer $\mathbf{A}$ consists of three 3,6-dialkynyl phenanthrene units linked by phosphodiesterbridges, which was synthesized by solid phase synthesis. ${ }^{[5 d]}$

The light-harvesting fibers were assembled from an aqueous solution of oligomer $\mathbf{A}$ by supramolecular polymerization. The aqueous solution is heated to $80^{\circ} \mathrm{C}$, which leads to the disassembly of the oligomer A building blocks. Afterwards, the solution is cooled to $20^{\circ} \mathrm{C}$ over ten minutes, which causes the building blocks to start assembling again and form the supramolecular polymer fibers due to hydrophobic interactions (see the Supporting Information).$^{[5 d]}$ Different artificial LHCs were obtained by performing the above procedure in the presence of the various acceptors. The following chromophores were tested as acceptors, uncharged ones: BaP, DAP, Cor, FA, Per; and charged ones: R6G, AO, EtBr (Figure 1). The different acceptors were added to oligomer $\mathbf{A}$ in increasing amounts. $\mathbf{A}$ heating/cooling cycle was performed after addition of the chromophores, to ensure a complete incorporation of the acceptor into the fibers. As verified by atomic force microscopy (AFM, Figure 4), the presence of the acceptors had no noticeable effect on the morphology of the supramolecular fibers at the concentrations tested. 
Figure 2 shows an illustration of the self-assembly process of trimer $\mathbf{A}$ and incorporation of an acceptor chromophore (benzo(a)pyrene, shown in red) into the growing supramolecular polymer. Upon cooling, A and the acceptor co-assemble into the fiber. The illustration displays an intercalative association between trimer $\mathbf{A}$ and the acceptor. Other modes of interaction (e.g. due to electrostatic interaction between phosphate groups and the positive charges of cationic acceptors) are well possible and are, thus, not ruled out. The different binding modes could affect the light-harvesting properties. This was already shown that by changing the linker-geometry of the phenanthrenes and thus the transition dipole moments, the light-harvesting efficiency is affected. ${ }^{[3 b, 5 d]}$

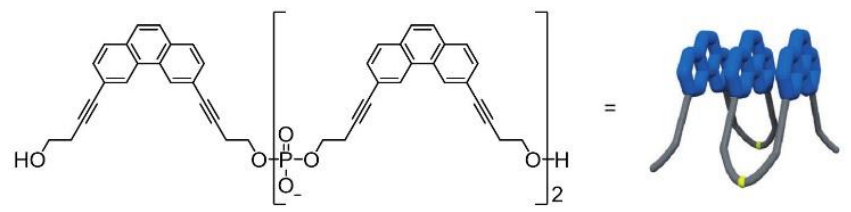

oligomer $\mathbf{A}$

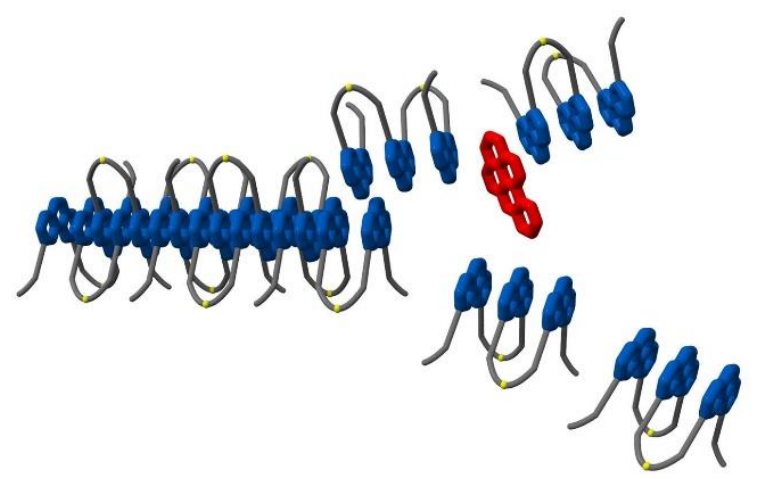

assembly $\downarrow$ disassembly

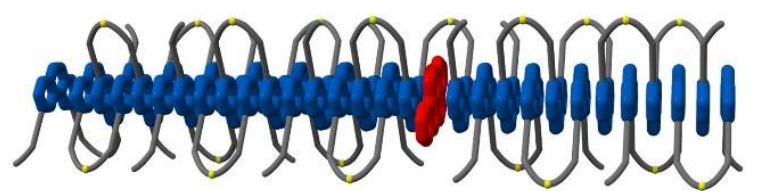

Figure 2. Illustration of an idealized assembly of a supramolecular polymer of oligomer A (blue) and a chromophore (benzo(a)pyrene, red), which is randomly integrated into the forming supramolecular polymer.

Figure 3 (left side) shows the fluorescence spectra of the artificial LHC formed from oligomer $\mathbf{A}$ in the absence or presence of three different chromophores: benzo(a)pyrene (BaP), 9,10-diphenylanthracene (DPA) and acridine orange (AO). The supramolecular polymer of oligomer $\mathbf{A}$ alone exhibits only weak fluorescence (black curves), upon excitation of Phe at $322 \mathrm{~nm}$. After addition of an acceptor chromophore, the fluorescence spectrum completely changes. While the emission of $\mathbf{A}$ is still present, a much more intense emission of the added chromophore appears. The respective curves show the effect of different acceptor/Phe ratios. Figure $3 \mathrm{~A}$ shows mainly the emission of BaP (maxima at $406 \mathrm{~nm}, 430 \mathrm{~nm}$ and $457 \mathrm{~nm}$ ). The Phe emission is still visible in the presence of BaP. Similar results were obtained by addition of DPA (Figure 3B, maxima at $411 \mathrm{~nm}$ and $431 \mathrm{~nm}$ ) or of $\mathbf{A O}$ (Figure $3 C$, maximum at $530 \mathrm{~nm}$ ) to oligomer $\mathbf{A}$. In the latter case, however, the Phe emission at $403 \mathrm{~nm}$ is decreasing and the $\mathbf{A O}$ emission is increasing by the addition of $\mathbf{A O}$ (blue and orange curves). The fact that the emission of the donor decreases while that of the acceptor increases suggests that energy transfer occurs at least partially via a FRET mechanism. In the other two cases, the LHCs with BaP and DPA, no or only a small decrease in the Phe emission is observed along with a large fluorescence band from BaP and DPA emission. This behavior is a sign that also energy transfer mechanisms other than FRET are involved, e.g. coherent energy transfer. ${ }^{[7]}$ Already a small addition of $1 \%$ of acceptor chromophore lead to significant fluorescence intensities. This is impressively illustrated by the development of the fluorescence quantum yields ( $\Phi_{F}$, Figure 3 ) upon addition of the acceptors. The fluorescence quantum yield is increasing in a non-linear fashion until it reaches a plateau (Figure $3 \mathrm{~A}$ and $\mathrm{B}$ ). When having a look at the charged acceptor $\mathbf{A O}$ (Figure $3 \mathrm{C}$ ), one can observe a decrease in $\Phi_{F}$ when reaching its maximum. This phenomenon was only observed when adding a charged chromophore. The reason for this is probably that after a certain concentration, the chromophores start to bind differently to the fibers than before and thus lead to a decrease in fluorescence quantum yield. These data confirm that acceptor-doped fibers are formed, although the type of molecular interaction cannot be derived from the fluorescence data. Nevertheless, the interaction allows efficient energy transfer and results in a complex with very good light-harvesting effects ( $\Phi_{\mathrm{F}}$ up to $\left.30 \%\right)$.

A)
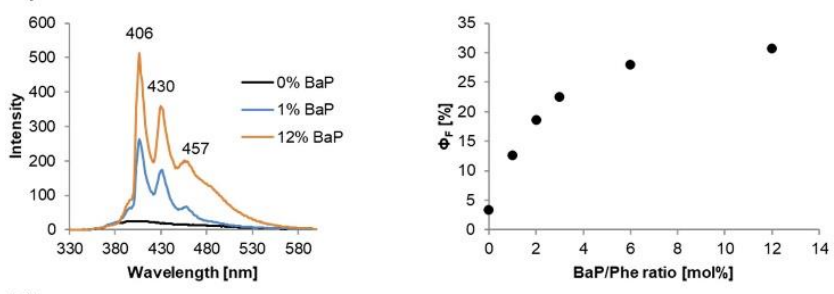

B)
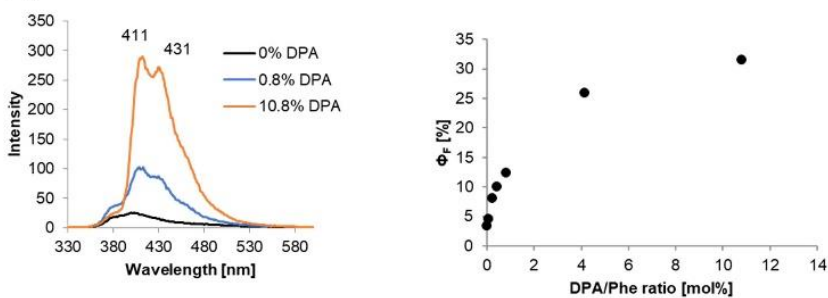

C)
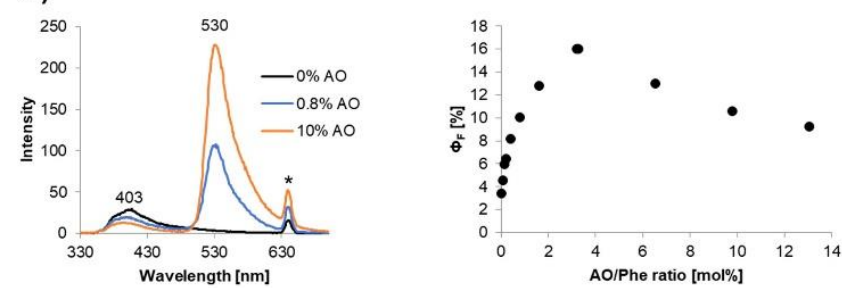

Figure 3. Left: Fluorescence spectra of the assembled supramolecular polymer in absence (black) and in the presence of the acceptor chromophores $\operatorname{BaP}(A)$, DAP $(B)$ and AO (C). Right: Fluorescence quantum yields $\left(\Phi_{F}\right)$ of the light-harvesting supramolecular fibers as a function of the acceptor/Phe ratio. Conditions: oligomer A $0.5 \mu \mathrm{M}, 10 \mathrm{mM}$ sodium phosphate buffer, $\mathrm{pH} 7.2, \lambda_{\mathrm{ex}}=$ $322 \mathrm{~nm}$. ( ${ }^{*}$ indicates a second-order diffraction) 
Next to the three molecules presented above, five additional chromophores also showed acceptor properties. Three of these (Cor, FA and Per), also uncharged molecules, revealed a slightly lower fluorescence quantum yield than BaP and DPA (Table 1). Of the remaining three-positively charged chromophores that were tested, R6G has the highest fluorescence quantum yield. Somewhat surprisingly, ethidium bromide showed no increase in fluorescence $\left(\Phi_{\mathrm{F}}=3.1 \%\right)$ compared to the Phe antenna itself $\left(\Phi_{F}=3.4 \%\right)$.

Table 1. The tested chromophores and their fluorescence quantum yields, compared to the $\Phi_{\mathrm{F}}$ of oligomer $\mathbf{A}(3.4 \%)$.

\begin{tabular}{lc}
\hline Chromophore & $\begin{array}{c}\text { Fluorescence Quantum Yield }\left(\Phi_{\mathrm{F}}\right) \text { at } \\
\mathrm{a} \sim 1 \text { mol\% acceptor/Phe ratio }[\%]^{[\mathrm{a}]}\end{array}$ \\
\hline Benzo(a)pyrene (BaP) & $12.6^{[\mathrm{c}]}$ \\
9,10-Diphenylanthracene (DPA) & $12.4^{[\mathrm{b}]}$ \\
coronene (Cor) & $6.7^{[\mathrm{b}]}$ \\
fluoranthene (FA) & $5.8^{[\mathrm{c}]}$ \\
perylene (Per) & $4.0^{[\mathrm{b}]}$ \\
rhodamine 6G (R6G) & $14.3^{[\mathrm{d}]}$ \\
acridine orange (AO) & $10.1^{[\mathrm{b}]}$ \\
ethidium bromide (EtBr) & $3.1^{[\mathrm{e}]}$ \\
\hline
\end{tabular}

${ }^{[\mathrm{a}]}$ Supporting Information, ${ }^{[\mathrm{b}]} 0.8 \mathrm{~mol} \%,{ }^{[\mathrm{c}]} 1 \mathrm{~mol} \%,{ }^{[\mathrm{d}]} 1.1 \mathrm{~mol} \%,{ }^{[\mathrm{e}]} 1.2 \mathrm{~mol} \%$

AFM images of the supramolecular polymer fibers are shown in Figure 4. They confirm that the presence of an acceptor chromophore (here $1 \%$ of DPA or $1 \%$ AO) does not alter the morphology of the fibers. The height and length of the formed fibers are in the same range as without any acceptor. The deflection scans (Figure 4B, D and F) show that the fibers are twisted together and cross each other on the mica surface. The self-assembly process is, thus, not affected by the presence of the acceptor chromophore.

In conclusion, we have demonstrated the formation of artificial LHCs by doping supramolecular Phe fibers with different types of acceptor chromophores. Energy transfer from the Phe fiber (donor) to the acceptor chromophores was observed with all tested chromophores. Furthermore, addition of the acceptor generally resulted in an increased fluorescence quantum yield; except in one case, EtBr, the quantum yield was comparable to the one of the Phe fibers alone. The fluorescence quantum yield of the fibers doped with $1 \mathrm{~mol} \%$ of $\mathrm{BaP}$ was highest (12.6\%) among the uncharged chromophores. Of the cationic chromophores, R6G exhibited the highest fluorescence quantum yield $(14.3 \%)$ at $1.1 \mathrm{~mol} \%$ doping. The results show that supramolecular Phe fibers act as light-harvesting antennae for excitation of various types of cationic and uncharged aromatic chromophores in aqueous environment. AFM measurements showed that doping with acceptor molecules had no effect on the morphology of the supramolecular polymer fibers by them. Phosphodiester-linked Phe oligomers as the one shown here are interesting as materials for amplification of diagnostic signals, for detecting polyaromatic pollutants in water or as components for artificial LHCs.
A)

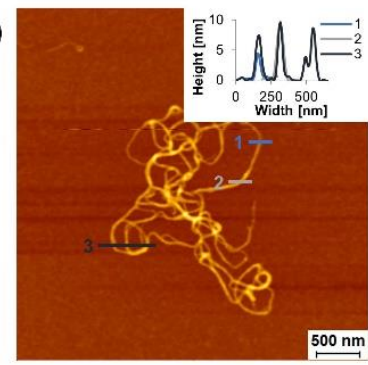

B)

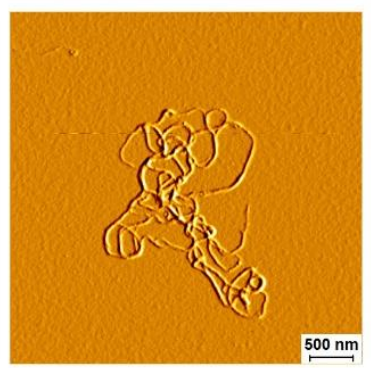

C)

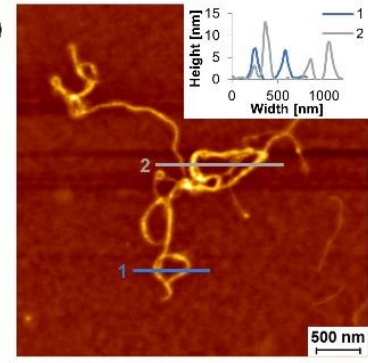

D)

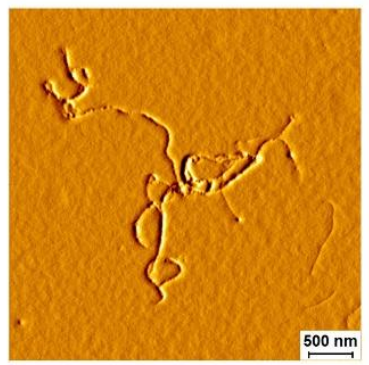

E)

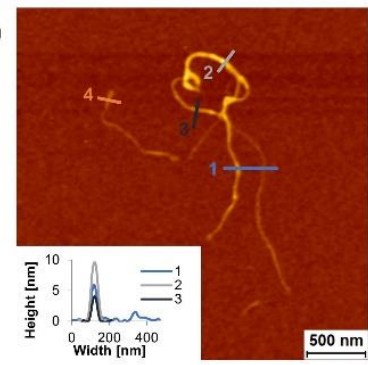

F)

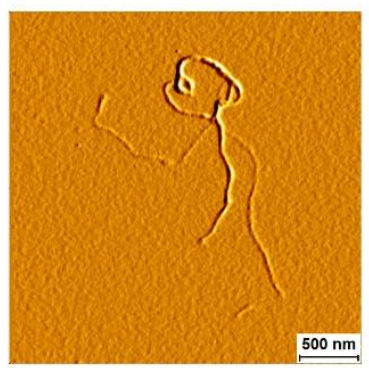

Figure 4. AFM pictures of supramolecular polymer fibers formed from oligomer $\mathbf{A}(\mathrm{A})$ (conditions as in Figure 3 ) in the presence of $1 \%$ DPA (C) or $1 \%$ AO (E) after deposition on APTES-modified mica; inset: height profiles as indicated in the image. (B), (D) and (F) depict the deflection scans of the corresponding AFM image on the left side.

\section{Acknowledgements}

Financial support by the Swiss National Foundation (grant 200020_188468) is gratefully acknowledged.

Keywords: energy transfer $\cdot$ light-harvesting antenna $\bullet \pi-\pi$ interactions $\bullet$ supramolecular polymers $\bullet$ phosphodiester

a) T. Xiao, W. Zhong, L. Zhou, L. Xu, X. Q. Sun, R. B. P. Elmes, X. Y. Hu, L. Wang, Chinese Chem. Lett. 2019, 30, 31-36; b) K. V. Rao K. K. R. Datta, M. Eswaramoorthy, S. J. George, Chem. - A Eur. J. 2012, 18, 2184-2194; c) T. Brixner, R. Hildner, J. Köhler, C. Lambert, F. Würthner, Adv. Energy Mater. 2017, 7, 1-33; d) A. Maity, A. Dey, M. Gangopadhyay, A. Das, Nanoscale 2018, 10, 1464-1473; e) C. L. Sun, H. Q. Peng, L. Y. Niu, Y. Z. Chen, L. Z. Wu, C. H. Tung, Q. Z. Yang, Chem. Commun. 2018, 54, 11171120; f) Y. Shi, X. Cao, D. Hu, H. Gao, Angew. Chemie 2018, 130, 525-529; g) J. J. Li, Y. Chen, J. Yu, N. Cheng, Y. Liu, Adv. Mater. 2017, 29, 1-5; h) C. Li, J. Zhang, S. Zhang, Y. Zhao, Angew. Chemie - Int. Ed. 2019, 58, 1643-1647; i) S. Guo, Y. Song, Y. He, X.-Y. Hu, L. Wang, Angew. Chemie 2018, 130, 3217-3221.

[2] a) H. Q. Peng, L. Y. Niu, Y. Z. Chen, L. Z. Wu, C. H. Tung, Q. Z. Yang, Chem. Rev. 2015, 115, 7502-7542; b) M. Surin, Polym. Chem. 2016, 7, 4137-4150; c) E. Magdalena Estirado, M. A. 
Aleman Garcia, J. Schill, L. Brunsveld, J. Am. Chem. Soc. 2019,

141, 18030-18037; d) J. G. Woller, J. K. Hannestad, B. Albinsson, J. Am. Chem. Soc. 2013, 135, 2759-2768; e) E. Krieg, M. M. C. Bastings, P. Besenius, B. Rybtchinski, Chem. Rev. 2016, 116 , 2414-2477; f) E. Boulais, N. P. D. Sawaya, R. Veneziano, A. Andreoni, J. L. Banal, T. Kondo, S. Mandal, S. Lin, G. S. SchlauCohen, N. W. Woodbury, H. Yan, A. Aspuru-Guzik, M. Bathe, Nat. Mater. 2018, 17, 159-166; g) N. Appukutti, C. J. Serpell, Polym. Chem. 2018, 9, 2210-2226; h) A. Ruiz-Carretero, P. G. A. Janssen, A. Kaeser, A. P. H. J. Schenning, Chem. Commun. 2011, 47, 43404347 ; i) C. D. Bösch, J. Jevric, N. Bürki, M. Probst, S. M. Langenegger, R. Häner, Bioconjug. Chem. 2018, 29, 1505-1509; j) F. J. M. Hoeben, P. Jonkheijm, E. W. Meijer, A. P. H. J. Schenning, Chem. Rev. 2005, 105, 1491-1546.

[3] a) F. Garo, R. Häner, Angew. Chemie - Int. Ed. 2012, 51, 916-919; b) C. D. Bösch, S. M. Langenegger, R. Häner, Angew. Chemie - Int. Ed. 2016, 55, 9961-9964; c) M. Kownacki, S. M. Langenegger, S. X. Liu, R. Häner, Angew. Chemie - Int. Ed. 2019, 58, 751-755.

[4] a) Y. Zhu, B. Yang, S. Chen, J. Du, Prog. Polym. Sci. 2016, 1-22; b) X. Li, N. Markandeya, G. Jonusauskas, N. D. McClenaghan, V. Maurizot, S. A. Denisov, I. Huc, J. Am. Chem. Soc. 2016, 138, 13568-13578; c) A. T. Haedler, K. Kreger, A. Issac, B. Wittmann, M. Kivala, N. Hammer, J. Köhler, H. W. Schmidt, R. Hildner, Nature 2015, 523, 196-199; d) T. Mashima, K. Oohora, T. Hayashi, Phys. Chem. Chem. Phys. 2018, 20, 3200-3209; e) V. L. Malinovskii, A. L. Nussbaumer, R. Häner, Angew. Chemie - Int. Ed. 2012, 51, 49054908.

[5] a) A. Ustinov, H. Weissman, E. Shirman, I. Pinkas, X. Zuo, B. Rybtchinski, J. Am. Chem. Soc. 2011, 133, 16201-16211; b) M. R. Wasielewski, Acc. Chem. Res. 2009, 42, 1910-1921; c) M. Hao, G. Sun, M. Zuo, Z. Xu, Y. Chen, X.-Y. Hu, L. Wang, Angew. Chemie Int. Ed. 2019, 58, 2-8; d) C. B. Winiger, S. Li, G. R. Kumar, S. M. Langenegger, R. Häner, Angew. Chemie - Int. Ed. 2014, 53, 1360913613; e) W. P. Klein, B. S. Rolczynski, S. M. Oliver, R. Zadegan, S. Buckhout-White, M. G. Ancona, P. D. Cunningham, J. S. Melinger, P. M. Vora, W. Kuang, I. L. Medintz, S. A. Díaz, ACS Appl. Nano Mater. 2020, 3, 3323-3336.

[6] M. Nazari, C. D. Bösch, A. Rondi, A. Francés-Monerris, M. Marazzi, E. Lognon, M. Gazzetto, S. M. Langenegger, R. Häner, T. Feurer, A. Monari, A. Cannizzo, Phys. Chem. Chem. Phys. 2019, 21, 1698116988.

A. Chenu, G. D. Scholes, Annu. Rev. Phys. Chem. 2015, 66, 69-96. 


\section{Entry for the Table of Contents}

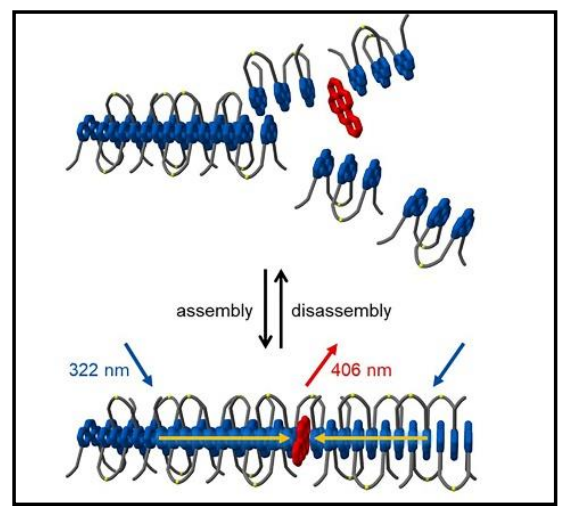

Supramolecular polymers formed from phosphodiester-bridged phenanthrene trimers are doped with charged and uncharged acceptor chromophores. Excitation of the phenanthrenes is followed by efficient energy transfer to the acceptor molecules.

Key Topic: Light-harvesting Complex

Institute and/or researcher Twitter usernames: @DCBunibern 